Check for updates

Cite this: RSC Adv., 2017, 7, 37148

Received 19th May 2017

Accepted 14th July 2017

DOI: $10.1039 / \mathrm{c} 7 \mathrm{ra05667b}$

rsc.li/rsc-advances

\section{Improved electromechanical properties of brominated butyl rubber filled with modified barium titanate $\uparrow$}

\author{
Mengnan Ruan, (D) ac Dan Yang, ${ }^{\text {tbd }}$ Wenli Guo, ${ }^{\text {abc }}$ Shuo Huang, ${ }^{\text {ac }}$ Yibo Wu, ${ }^{\text {bc }}$ \\ Hao Wang, ${ }^{\text {bc Huaming Wang }}{ }^{d}$ and Liqun Zhang (D)
}

In order to improve the compatibility between barium titanate (BT) particles and a brominated butyl rubber (BIIR) matrix, a silane coupling agent $\gamma$-methacryloxy propyl trimethoxysilane ( $\mathrm{KH} 570$ ) was used to modify the BT particles. Subsequently, BT and the BT-KH570 particles were added into the BIIR matrix to form BT/ BIIR and BT-KH570/BIIR dielectric composites. The BT-KH570/BIIR composite has a higher dielectric constant, better mechanical properties, and larger actuated strain compared with the BT/BIIR composite with the same filler content. The $10 \mathrm{phr}$ BT-KH570/BIIR composite achieved the largest actuated strain. To further increase the actuated strain of the BT-KH570/BIIR composite, the polar plasticizer tri- $n$-butyl phosphate (TBP) was added into the composite. Due to the polar groups of TBP, the dielectric constant of the TBP/BT-KH570/BIIR composite was improved and the elastic modulus decreased at the same time. As a result, we obtained a gain in actuated strain of $4.46 \%$ at an electric field of $22.5 \mathrm{kV} \mathrm{mm}^{-1}$, which corresponds to an increase of about $500 \%$ in comparison with that of pure BIIR. This represents a significant improvement in actuated strain performance.

\section{Introduction}

Electroactive polymers (EAPs), which can change their shape under an electrical field, are commonly studied for their possible use in mimicking muscle-like polymers. ${ }^{1-3}$ In the field of EAPs, dielectric elastomers (DEs) are widely used in aerospace, biomedical fields, energy harvesting, flexible electronics, and smart actuators due to their large active strain, fast response, and simple working principle. ${ }^{4}$ Dielectric elastomer actuators (DEAs) are made by spraying compliant electrodes on two sides of elastic thin films. Corresponding to the advantages associated with a simple working principle, low cost, and large deformation, DEAs have exceptional prospects for use in electric fields. ${ }^{5}$

The actuated stress $(p)$ and actuated strain $\left(s_{z}\right)$ of the dielectric elastomer actuators (DEAs) are determined as follows:

$$
\begin{gathered}
p=\varepsilon_{0} \varepsilon_{\mathrm{r}} E^{2} \\
s_{z}=-\frac{p}{Y}=-\frac{\varepsilon_{0} \varepsilon_{\mathrm{r}} E^{2}}{Y}
\end{gathered}
$$

${ }^{a}$ College of Materials Science and Engineering, Beijing University of Chemical Technology, Beijing 100029, China.E-mail: gwenli@bipt.edu.cn

${ }^{b}$ College of Materials Science and Engineering, Beijing Institute of Petrochemical Technology, Beijing, 102617, China.E-mail: yangdan@bipt.edu.cn

${ }^{c}$ Beijing Key Lab of Special Elastomeric Composite Materials, Beijing, 102617, China ${ }^{d}$ College of Mechanical and Electrical Engineering, Nanjing University of Aeronautics and Astronautics, Nanjing, 210016, China

$\dagger$ Electronic supplementary information (ESI) available. See DOI: 10.1039/c7ra05667b where $\varepsilon_{0}$ and $\varepsilon_{\mathrm{r}}$ are the vacuum permittivity and the dielectric constant of the DEs, respectively. $E$ is the applied electric field, and $Y$ is the elastic modulus of the material. From eqn (2), we can find that the actuated strain can be improved by increasing the permittivity and decreasing the modulus of the dielectric materials, meaning a high electromechanical sensitivity $\beta=\varepsilon / Y$ is needed.

Dielectric elastomer materials, which affect the size of the actuated strain directly, are the basis of DEAs. ${ }^{6}$ Commonly used dielectric elastomer materials include silicone elastomers, polyurethane, acrylate, and poly(vinylidene fluoride) (PVDF). Of these materials, silicone elastomers are widely used as DEs due to its favorable abilities, such as good elasticity, fast response speed, and good stability. ${ }^{7,8}$ However, the silicone elastomer, which is non-polar, has a low dielectric constant less than $3 .^{9}$ Moreover, silicone elastomers also require a high bias voltage due to the low energy density, which limits its application for use as actuators. Polyurethane (PU) consists of strong polar groups and exhibits high flexural endurance, high shape recoverability, and good biocompatibility, which can be used for flexible micro-actuators. ${ }^{\mathbf{1 0}}$ However, the elastic modulus of PU is relatively high, which leads to a low actuated strain. The common acrylic elastomer VHB4910 (3M), which is widely applied in DE actuators, has the properties of low cost, good mechanical performance, good compatibility, and is easy to process. However, due to the large stickiness, VHB4910 has a serious hysteresis phenomenon, which leads to generation of leakage current and energy loss. ${ }^{11,12}$ PVDF consisting of $-\mathrm{CH}_{2}-$ 
$\mathrm{CF}_{2}$ - repetitive unit is a semi-crystalline polymer with good chemical stability, high piezoelectric coefficients and toughness, and a stable thermal performance. However, due to its semi-crystalline structure, PVDF has a high elastic modulus, which limits its electro-deformation performance in an electric field. $^{13-15}$

Essentially, only few commercially homogeneous DE films can be applied for use as a dielectric elastomer. Several researchers tend to prepare and find new DE materials with both high dielectric constant and low elastic modulus.. ${ }^{16,17}$ Yeonju Jang et $a l^{18}$ investigated poly(methylmethacrylate) (PMMA)-b-poly(nbutylacrylate) (PnBA)-b-poly(methylmethacrylate) (PMMA) films with cylindrical and lamellar morphologies, which were prepared with toluene and acetone casting solvents. The maximum strain of the toluene PMMA-PnBA-PMMA film actuator was almost $12 \%$, about 3 times larger than an acetone PMMA-PnBA-PMMA film actuator. Haibin Sun et al. ${ }^{19}$ prepared a new homogeneous styrene-butadiene-styrene triblock copolymer (SBS) dielectric elastomer grafted with dipoles (ester groups) on it. The modified SBS had higher dielectric constant and larger actuated strain than the stock SBS. Kie Yong Cho et al. ${ }^{20}$ obtained a hard-soft-hard triblock-random copolymers (PTMDMT), which consisted of hard poly(methylmethacrylate) (PMMA) and soft poly(dodecyl methacrylate) (PDMA). The PTMDMT copolymers showed a transverse

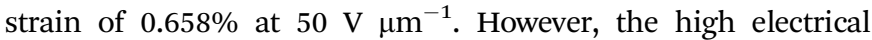
breakdown field, which is harmful for the human body, limited their development in dielectric elastomer materials. In conclusion, these methods are environmentally unfriendly and need multi-step procedures and complex instruments.

Brominated butyl rubber (BIIR) has numerous advantages, such as heat resistance, ozone resistance, chemical corrosion resistance, high dielectric hysteresis, flex high fatigue strength, and low permeability, and can be used as a candidate dielectric matrix. In this study, we choose BIIR as the dielectric elastomer material for the first time. ${ }^{21}$ In order to improve the dielectric constant of BIIR, the high-dielectric-constant fillers are usually added into polymeric matrix to form dielectric elastomer composites. As the barium titanate (BT) particles are low cost, environmentally friendly, and have a dielectric constant as high as 1700 at room temperature, we choose ferroelectric BT nanoparticles as a dielectric filler to improve dielectric properties of BIIR. Various researchers mixed the BT particles into a polymer matrix to improve the dielectric properties of the polymer matrix. For example, Tao Zhou et al. ${ }^{22}$ prepared poly(vinylidenefluoride) (PVDF) based nanocomposites by adding surface hydroxylated $\mathrm{BaTiO}_{3}$ (h-BT) nanoparticles into PVDF. The h-BT/PVDF composites showed lower loss tangent and higher dielectric strength BT/PVDF composites. Zhimin Dang et $a .^{23}$ prepared $\mathrm{BT} / \mathrm{PVDF}$ nanocomposites via a natural adsorption action occurring between the nanosized BT and PVDF particles in absolute ethanol and subsequent hot-press step. The results showed that the homogeneous BT/PVDF composites displayed desirable dielectric properties with a high dielectric constant of 45 at $1 \mathrm{kHz}$. Liang Jiang et al. ${ }^{24}$ fabricated a new soft dielectric elastomer composite by adding dopamine coated bariumtitanate (DP-BT) particles into silicone rubber (SR). It was found that the dielectric constant increased and dielectric loss tangent decreased in SR/DP-BT composites compared with those of the SR/BT composites. However, in order to improve compatibility between BT and BIIR, the silane coupling agent $\gamma$-methacryloxypropyl trimethoxysilane (KH570) was used to modify BT in our study. The BT-KH570/BIIR composites were prepared by physical blending, which was easy to perform and was harmless to the human body. In previous studies, Zhimin Dang et al. ${ }^{25}$ prepared a $\mathrm{TiO}_{2} /$ silicone rubber (SR) nanocomposite via three-roll grinding followed compressive molding technology. The $\mathrm{TiO}_{2}$ nanoparticles were chemically modified with KH570. The results showed that electrical breakdown strength and tensile strength of $\mathrm{TiO}_{2}{ }^{-}$ KH570/SR enhanced while the dielectric loss decreased relative to $\mathrm{TiO}_{2} / \mathrm{SR}$. The results also indicated that the uniform dispersion of $\mathrm{TiO}_{2}-\mathrm{KH} 570$ particles enhanced interfacial interaction between $\mathrm{TiO}_{2}$ and SR matrix. Dan Yang et al. ${ }^{26}$ prepared a slide-ring material with a necklace-like molecular structure as dielectric elastomer materials. BT particles modified by KH570 were added into the SR materials to further increase the actuated performance. The results showed that an actuated strain $(26 \%)$ at a low electric field $\left(12 \mathrm{kV} \mathrm{mm}^{-1}\right)$ was obtained on the circular membrane actuator without any pre-strains. In our study, to further improve the actuated strain of BT-KH570/BIIR composites, the polar tri- $n$-butyl phosphate (TBP) plasticizer was added into BT-KH570/BIIR composites to decrease the elastic modulus of the elastomer. The microstructure, mechanical properties, dielectric properties, and actuated strains of BIIR composites were then thoroughly investigated.

\section{Experiment}

\subsection{Materials}

Commercial brominated butyl rubber (BIIR) (BIIR 2255, mass fraction of bromine is $1.8 \%$, molecular weight is 550000 , product by Exxon, American) was used as the matrix. $\mathrm{BaTiO}_{3}$ particles with diameter of $30 \mathrm{~nm}$ were purchased from Beijing DK Nanotechnology Co., Ltd. (China). KH570, which is used as the coupling agent, and TBP were purchased from Beijing Chemical Reagents Co., Ltd. (China). Zinc oxide (ZnO) and calcium stearate (CaSt) were purchased from Beijing Societegenerale Honest Chemical Co., Ltd. (China).

\subsection{Modification of BT particles by KH570}

First, $100 \mathrm{ml}$ of ethanol was added to $900 \mathrm{ml}$ distilled water and mechanically stirred for 10 minutes. Then, $8 \mathrm{~g}$ KH570 was added into the solution with stirring for 20 minutes. Ammonia was added to $1000 \mathrm{ml}$ distilled water until the $\mathrm{pH}$ of solution was 11. These two solutions were mixed together and stirred for one hour. Second, $100 \mathrm{~g}$ BT particles were added to the abovementioned solution, and the solution was mechanically stirred for 6 hours. Then, the BT particles, which were modified with KH570 solutions, were washed with distilled water and then dried at $100{ }^{\circ} \mathrm{C}$ in a vacuum oven. Finally, all BT-KH570 particles were extracted for $36 \mathrm{~h}$ by refluxing ethanol in a Soxhlet extractor to remove the excess KH570. During extraction, the KH570, which were physically adsorbed, were washed by 
refluxing with ethanol. In addition, numerous researchers also indicated that the KH570 was chemically bonded onto the BT particles through a similar method to ours.

\subsection{Preparation of dielectric composites}

The uncured BIIR with different contents of BT, BT-KH570, and TBP were prepared by physical mixing on a 6 inch two-roll mill. Recipe (3.1) in our experiments contained different weight contents of BT or BT-KH570 (0 phr, 10 phr, 20 phr, 30 phr), 5 phr of $\mathrm{ZnO}, 1 \mathrm{phr}$ of CaSt and $100 \mathrm{~g}$ of BIIR, denoted as BT/BIIR or BTKH570/BIIR composites; Recipe (3.2) involved the addition of different weight contents of TBP (10 phr, $20 \mathrm{phr}, 30 \mathrm{phr}), 10 \mathrm{phr}$ BT-KH570 particles, 5 phr of ZnO, 1 phr of CaSt and $100 \mathrm{~g}$ of BIIR, which were denoted as TBP/BT-KH570/BIIR composites. Each composite was cured at the pressure of $15 \mathrm{MPa}$ at $160{ }^{\circ} \mathrm{C}$ for its optimum cure time as determined by a disk oscillating rheometer (GT-M2000-FA, Gotech Testing Machines Inc., Taiwan) to obtain the corresponding cured composite. The preparation process of TBP/BT-KH570/BIIR composites is shown in Fig. 1.

\subsection{Characterization methods}

XPS spectra of BT and BT-KH570 particles were recorded to study the elemental composition using an ESCALAB 250 XPS system, which was made in American Thermo Electron Corporation. In the XPS analysis, a monochromatic $\mathrm{Al} \mathrm{K \alpha} \mathrm{X}$-ray source was operation with pass energy of $1486.6 \mathrm{eV}$.

FTIR spectra were recorded on the German Company Bruker Tensor 27 infrared spectrometer. Samples of BT and BT-KH570 particles were ground and dispersed in $\mathrm{KBr}$, followed by compression to consolidate the formation of a pellet. FTIR spectra were obtained by the $\mathrm{KBr}$ pellet method at the wavenumber range of $600-4000 \mathrm{~cm}^{-1}$ at a resolution of $2 \mathrm{~cm}^{-1}$.

Thermogravimetric analysis (TGA) (TA Q500 thermogravimetric) was executed in the temperature range from $25{ }^{\circ} \mathrm{C}$ to $650{ }^{\circ} \mathrm{C}$ with a heating rate of $10{ }^{\circ} \mathrm{C} \min ^{-1}$ under nitrogen atmosphere.

Surface morphologies of BT/BIIR and BT-KH570/BIIR TBP/ BT-KH570/BIIR were characterized by FEI NanoSEM 430 scanning electron microscope.

The elastic modulus of the composites was tested by the slope of the stress-strain curve at 5\% strain, which were acquired using a tensile apparatus (RG2000-100, Shenzhen Reger. Instrument Co., Ltd., China) with crosshead speed of 50 $\mathrm{mm} \min ^{-1}$ at room temperature according to Chinese Standards GB/T 528-1998.

The dielectric properties of the composites were tested using an impedance analyzer (E4980A, Agilent, U.S.A.) over the frequency range of $10^{2}$ to $10^{6} \mathrm{~Hz}$ at $25^{\circ} \mathrm{C}$.

The actuated strain test was measured using a circular membrane actuator. Before measurement, two surfaces of the film were sprayed on graphite electrodes using an airbrush. Then, dielectric elastomer films were laid flat between two circular frames without any pre-strains. To get the largest strain, the voltage loaded on the electrode area was increased from $0 \mathrm{kV} \mathrm{mm}{ }^{-1}$ until an electric breakdown occurred, which is supplied by a high voltage power (73030P, Boher HV Co., Ltd.).

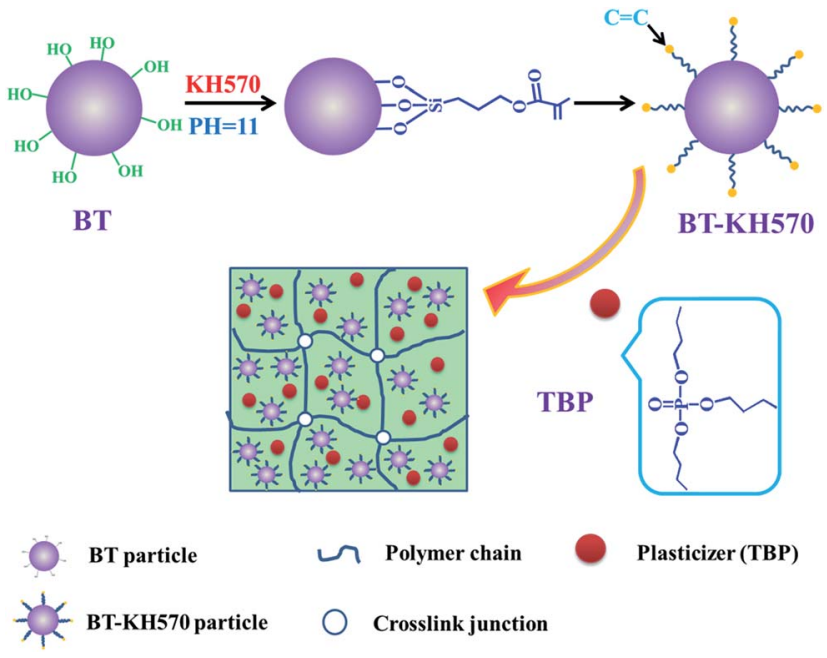

Fig. 1 The preparation process of TBP/BT-KH570/BIIR composites.

Then, we can compare the largest actuated stain obtained in our study with other dielectric elastomers easily. After we know the electric breakdown of dielectric elastomers, the electric field loaded on the dielectric elastomer actuator should be lower than their electric breakdown during practical application. During actuation, the video images of the biaxial extension of the electrode area film were captured by a camera (Canon Ixus 210, Japan) fitted with a wide-angle lens. We calculated the planar strain $\left(S_{\mathrm{p}}\right)$ by Adobe Photoshop software according to

$$
S_{\mathrm{p}}=\left(A-A_{0}\right) / A_{0} \times 100 \%
$$

where $A$ is the actuated planar area and $A_{0}$ is the planar area before actuation.

Every data point consisting of elastic modulus, stress-strain curve, dielectric properties, and actuated strain in this paper is the average value acquired from at least five samples under the same condition.

\section{Results and discussion}

\subsection{Effect of filler}

3.1.1 BT particles modified by KH570. The process and mechanism of surface modification are displayed in Fig. 2(a). XPS analysis of BT and BT-KH570 particles was employed to confirm the elemental content, and the results are shown in Fig. 2(b) and Table 1. From Fig. 2(b), we can find that there is a new peak component at a binding energy (BE) of about $104 \mathrm{eV}$ in BT-KH570 particles, which can be attributed to silicon in KH570. This affirms that the KH570 is grafted on BT particles. ${ }^{27}$

The ATR-FTIR spectra of BT and BT-KH570 particles are shown in Fig. $3 .{ }^{28}$ Compared with the BT particles, the BT-KH570 particles have new peaks appearing at $939 \mathrm{~cm}^{-1}$, $1039 \mathrm{~cm}^{-1}$, and $1725 \mathrm{~cm}^{-1}$, which represent $\mathrm{Si}-\mathrm{OH}, \mathrm{Si}-\mathrm{O}-\mathrm{C}$, and $\mathrm{C}=\mathrm{O}$, and the peaks appearing at $1129 \mathrm{~cm}^{-1}$ and 1167 $\mathrm{cm}^{-1}$, which represent $\mathrm{Si}-\mathrm{O}-\mathrm{Si}$ from $\mathrm{KH} 570$, respectively. In addition, the TGA curves of BT and BT-KH570 are shown in Fig. 4. From Fig. 4, we can find the BT and BT-KH570 particles 
(a)

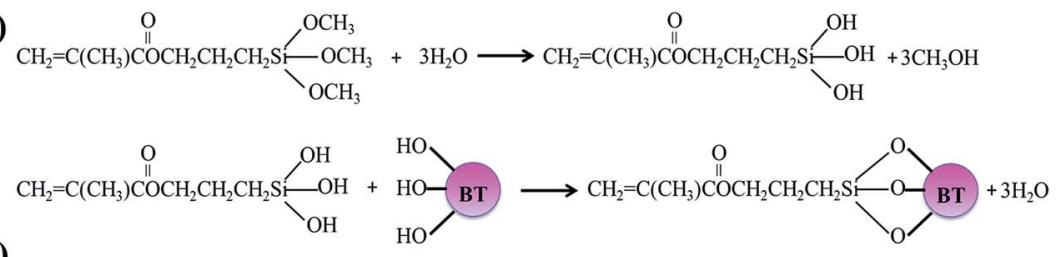

(b)

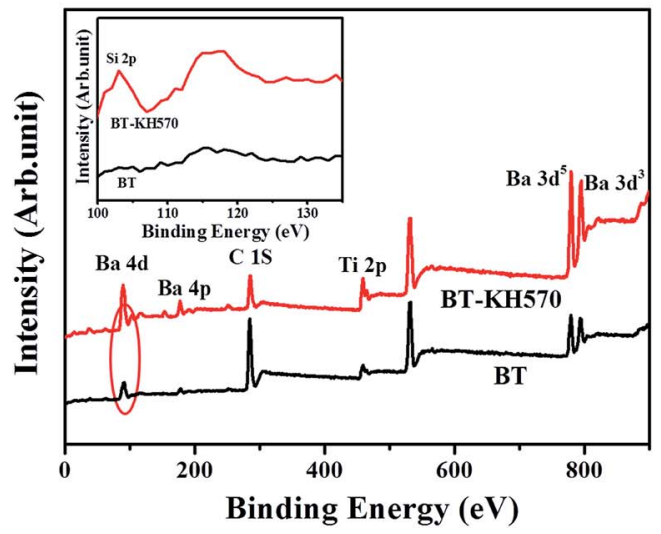

Fig. 2 (a) Schematic representation of BT particles modified with KH570. (b) X-ray photoelectron spectroscopy wide-scan spectra of BT and BTKH570 particles.

Table 1 Surface composition of BT and BT-KH570 particles by X-ray photoelectron spectroscopy

\begin{tabular}{llllll}
\hline & \multicolumn{5}{l}{ Element content $(\mathrm{wt} \%)$} \\
\cline { 2 - 6 } Samples & C 1s & O 1s & Ba 3d5 & Ti 2p & Si 2p \\
\hline BT & 69.8 & 25.92 & 1.98 & 2.29 & 0 \\
BT-KH570 & 39.17 & 37.87 & 6.54 & 6.07 & 10.36
\end{tabular}

showed a weight loss of $1.74 \%$ and $2.44 \%$, respectively, when samples were heated to $600{ }^{\circ} \mathrm{C}$. These results show that the KH570 is grafted to BT particles by chemical bonding instead of through physical adsorption.

3.1.2 Morphology of dielectric composites. The microstructures of transversally cut BT/BIIR and BT-KH570/BIIR composites were observed using SEM (Fig. 5). From Fig. 5(a) and (c), we can observe the agglomeration of BT particles, which occurs in a $10 \mathrm{phr}$ BT/BIIR and a $30 \mathrm{phr}$ BT/BIIR composite, which can be attributed to the large difference in surface energy of the BIIR matrix and BT particles. ${ }^{29}$ However, from Fig. 5(b) and (d), we can see that the BT-KH570 particles disperse uniformly in the BIIR matrix in spite of the filler content, which reaches 30 phr. Moreover, the interface between BT-KH570 particles and BIIR matrix is rather indistinct, which indicates good compatibility between the BT-KH570 particles and BIIR matrix. $^{24,30}$ In conclusion, the KH570 clearly improves the dispersion of BT/BIIR composites.

3.1.3 Mechanical properties of dielectric composites. The stress-strain curves of BT/BIIR and BT-KH570/BIIR composites are shown in Fig. 6(a) and (b). From Fig. 6(a) and (b), we can find that the tensile strength of the composites increases with increasing content of BT and BT-KH570 particles. However,

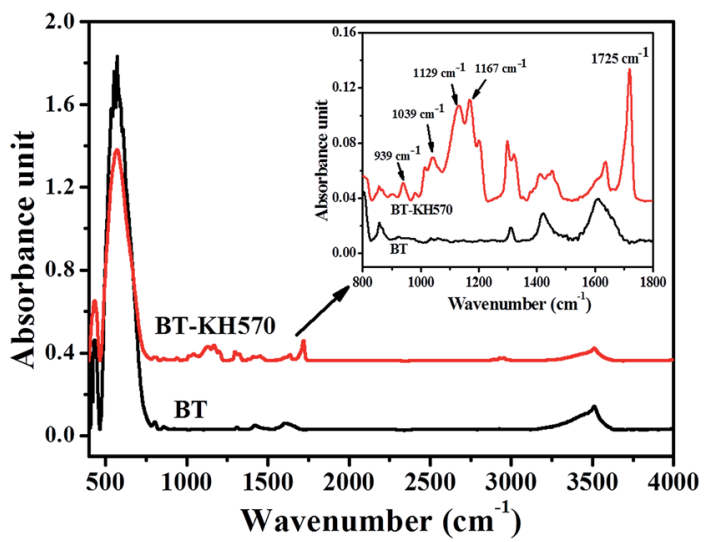

Fig. 3 The FTIR spectra of BT and BT-KH570 particles.

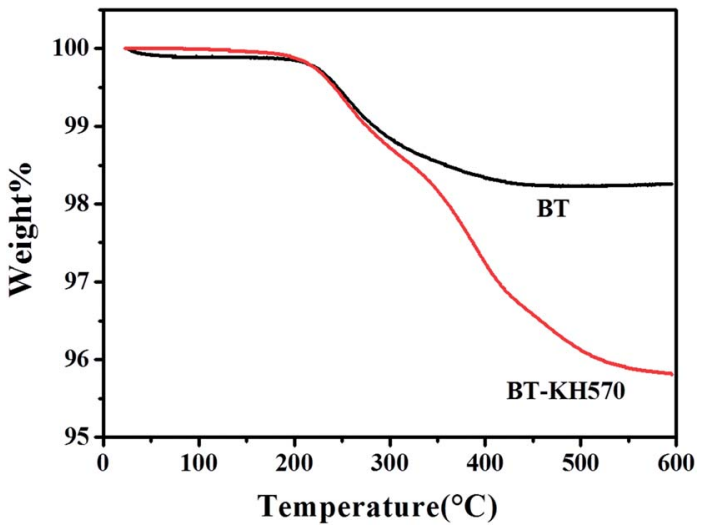

Fig. 4 TG curves of BT and BT-KH570 particles. 

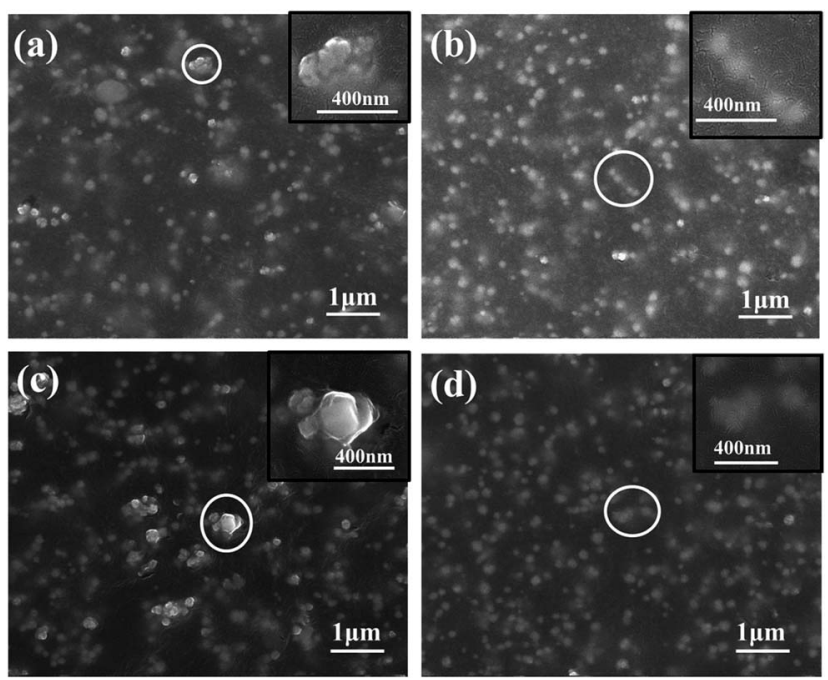

Fig. 5 SEM micrographs of fractured surfaces of BIIR composites filled with different contents of BT or BT-KH570 particles: (a) 10 phr of BT, (b) 10 phr of BT-KH570, (c) 30 phr of BT, (d) 30 phr of BT-KH570.

comparing with BT/BIIR composites, the BT-KH570/BIIR composites exhibit higher tensile strength at the same content of filler mainly because of the uniform dispersion of particles with less defects and strong interfacial interactions between the BT-KH570 particles and BIIR matrix. In addition, we can see that all composites show larger elongation at break than that of pure BIIR elastomer. It is possible that the crosslink density of the BIIR composites was decreased due to BT particles. $^{31}$ In addition, the elongation at break of BT-KH570/BIIR composites is always larger than that of BT/BIIR composites. This may be explained by the fact that the $\mathrm{C}=\mathrm{C}$ in $\mathrm{KH} 570$ is grafted into the BT particles, which participates in the crosslinking process and increases the elongation at break of BTKH570/BIIR composites. ${ }^{32,33}$

Fig. 6(c) shows the elastic modulus of BIIR composite filled with different contents of BT and BT-KH570 particles. From Fig. 6(c), we can see the elastic modulus of BIIR composite filled with 10 phr BT and BT-KH570 particles is the lowest over all range of filler content. The above phenomenon may be explained by two factors: the softening effect as a result of the interference on crosslinking and the hardening effect of the filler network. ${ }^{34}$ We can characterize the crosslink density of BIIR composites by the maximum torque $\left(S_{\max }\right)$ and minimum torque $\left(S_{\text {min }}\right)$ in the curing curve as shown in Table 2. From Table 2, we can find that the $\Delta H$ of BIIR composites is lower than that of pure BIIR, which can be attributed to the fact that BT particles decrease the crosslinking process of BIIR composites. The competing effects of crosslink density and filler network result in the lowest elastic modulus for the composite filled with 10 phr filler whether the BT particles are modified or not. In addition, from Fig. 6(c), we can also find that the elastic modulus of BT-KH570/BIIR composites is lower than that of BT/BIIR composites at the same filler content. This may be explained by two factors: one is that the $\Delta H$ (crosslink density) of BT-KH570/BIIR is lower than that of BT/BIIR composites (shown in Table 2). The other is that low
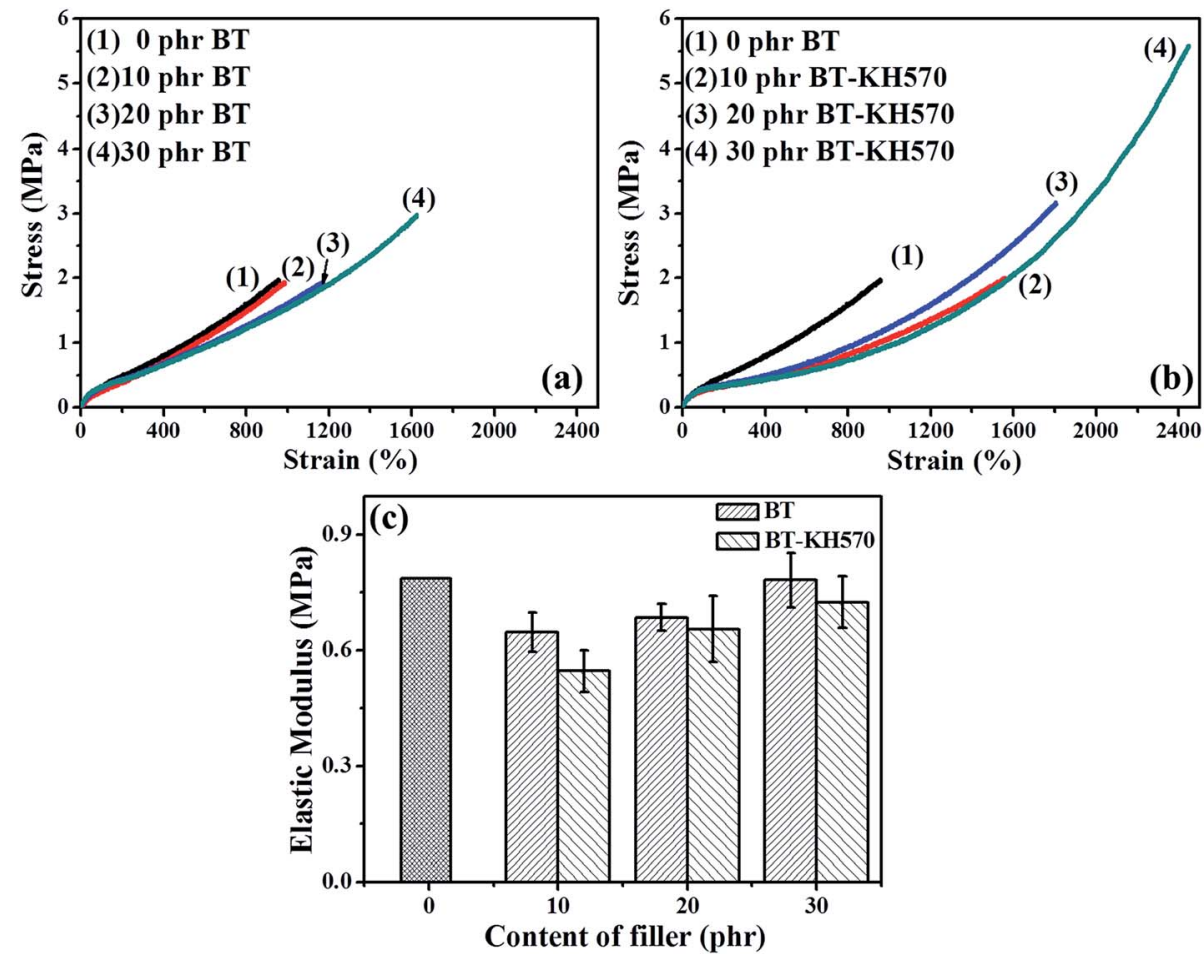

Fig. 6 Stress-strain curves of BIIR composites filled with different contents of (a) BT particles and (b) BT-KH570 particles. (c) Elastic modulus of BIIR composites filled with different contents of BT particles and BT-KH570 particles. 
Table 2 The maximum torque and the minimum torque of BIIR and BIIR with different contents of BT and BT-KH570

\begin{tabular}{llll}
\hline Samples & $\begin{array}{l}S_{\max } \\
(\mathrm{dN} \mathrm{m})\end{array}$ & $\begin{array}{l}S_{\min } \\
(\mathrm{dN} \mathrm{m})\end{array}$ & $\begin{array}{l}\Delta S \\
(\mathrm{dN} \mathrm{m})\end{array}$ \\
\hline BIIR & 5.49 & 1.27 & 4.22 \\
$10 \mathrm{phr} \mathrm{BT/BIIR}$ & 5.82 & 2.20 & 3.62 \\
$20 \mathrm{phr}$ BT/BIIR & 6.12 & 2.34 & 3.78 \\
$30 \mathrm{phr}$ BT/BIIR & 6.24 & 2.43 & 3.81 \\
$10 \mathrm{phr}$ BT-KH570/BIIR & 5.99 & 2.46 & 3.53 \\
$20 \mathrm{phr}$ BT-KH570/BIIR & 6.07 & 2.50 & 3.57 \\
$30 \mathrm{phr}$ BT-KH570/BIIR & 6.34 & 2.56 & 3.78
\end{tabular}

molecular KH570 grafted on the BT particles leads to a low elastic modulus of BT-KH570 particles. $^{35}$ Therefore, the elastic modulus of BT-KH570/BIIR composites is lower than that of BT/ BIIR composites.

3.1.4 Dielectric properties of dielectric composites. Dielectric properties of BT/BIIR and BT-KH570/BIIR composites at frequencies ranging from $10^{2} \mathrm{~Hz}$ to $10^{6} \mathrm{~Hz}$ are shown in Fig. 7. From Fig. 7(a) and (c), we can clearly see that the dielectric constant increased with increasing BT and BTKH570 content. In addition, we see that the dielectric constant of BT-KH570/BIIR composites is always higher than that of BT/BIIR composites over a broad frequency range. ${ }^{29,36}$ The improved dielectric constant of BT-KH570/BIIR composites can be explained by the good compatibility between the BT-KH570 particles and BIIR matrix, leading to a much improved interface polarization. Moreover, we can see that the dielectric constant remains almost constant with increasing frequency, thus demonstrating an advantage for use as a dielectric elastomer actuator. In addition, from Fig. 7(b) and (d), we find that the dielectric loss of BT/BIIR and BT-KH570/ BIIR composites is almost the same. However, the dielectric loss of BT/BIIR and BT-KH570/BIIR composites is less than 0.04 , meaning much electrical energy can be converted into mechanical energy. ${ }^{37}$

3.1.5 Actuated strain of dielectric composites. The relation between actuated strain and electric field for BT/BIIR and BTKH570/BIIR composites is shown in Fig. 8(a) and (b). From Fig. 8(a) and (b), we can find that all composites show higher actuated strains than that of pure BIIR. The highest actuated strain of $6.2 \%$ was obtained by $10 \mathrm{phr}$ BT-KH570/BIIR at $37.5 \mathrm{kV}$ $\mathrm{mm}^{-1}$, which is about 2 times higher than that of pure BIIR. ${ }^{24,37}$ According to the Maxwell equation, ${ }^{38,39}$ in order to obtain a superior actuated strain, the electromechanical sensitivity $(\beta)$, which is the ratio of dielectric constant to elastic modulus, is regarded as a significant value. When 10 phr filler was added into the BIIR matrix, the electromechanical sensitivity $(\beta)$ was the largest of the series, thus leading to the composites exhibiting the largest actuated strain. Whether BT/BIIR or BT-KH570/ BIIR composites, the electrical breakdown field increased with increasing content of fillers. This can be because the mobility of molecular chain was hindered when numerous particles were added into the matrix. It is difficult for the charges to move through the loose molecular chains, which lead to the improvement of breakdown strength. ${ }^{\mathbf{1 7 , 4 0 , 4 1}}$ Because of better filler dispersion and stronger interface interaction, the BTKH570/BIIR composites exhibit higher electric breakdown filed than that of BT/BIIR composites.
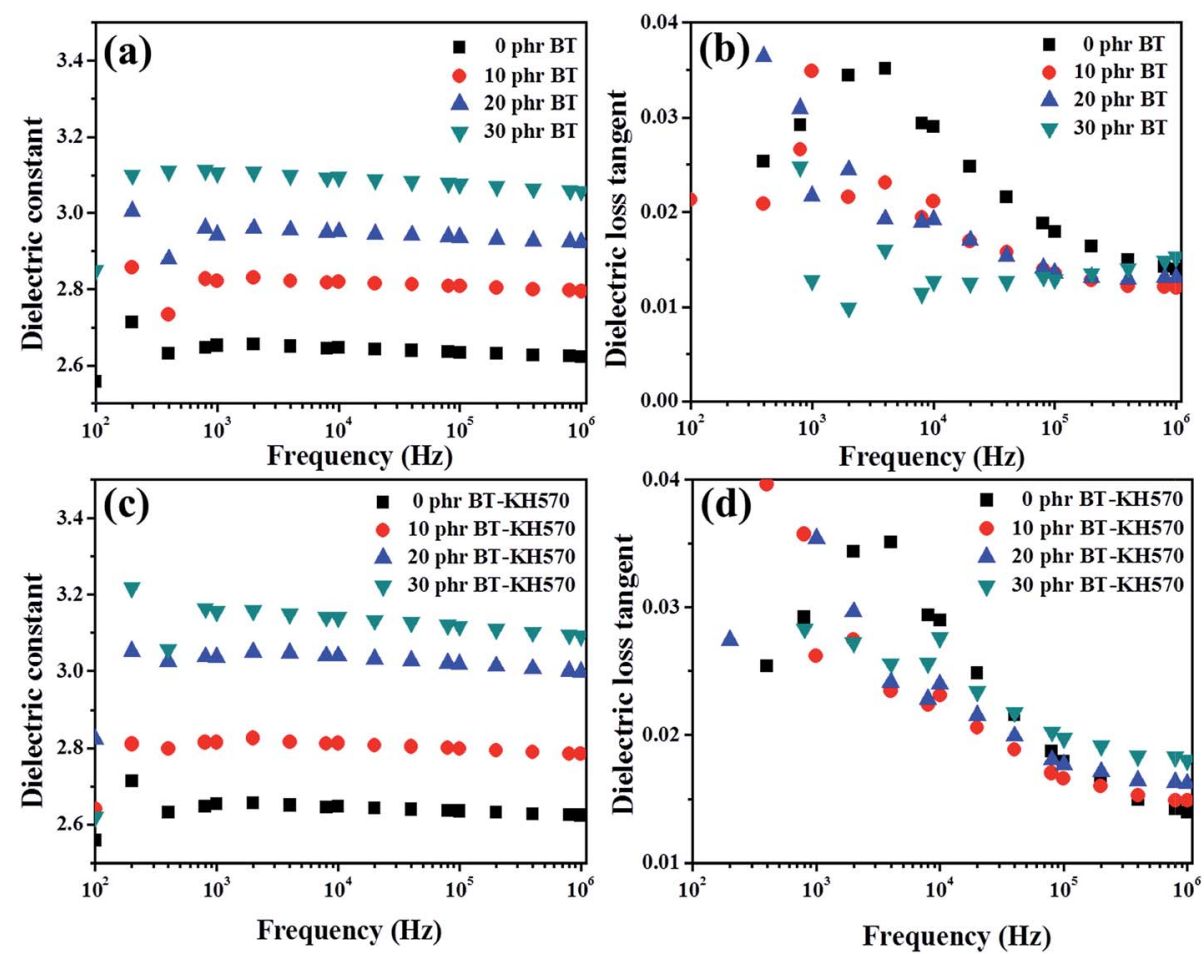

Fig. 7 Frequency dependence of (a) dielectric constant and (b) dielectric loss tangent of BIIR composites at different contents of BT particles. Frequency dependence of (c) dielectric constant and (d) dielectric loss tangent of BIIR composites at different contents of BT-KH570 particles. 

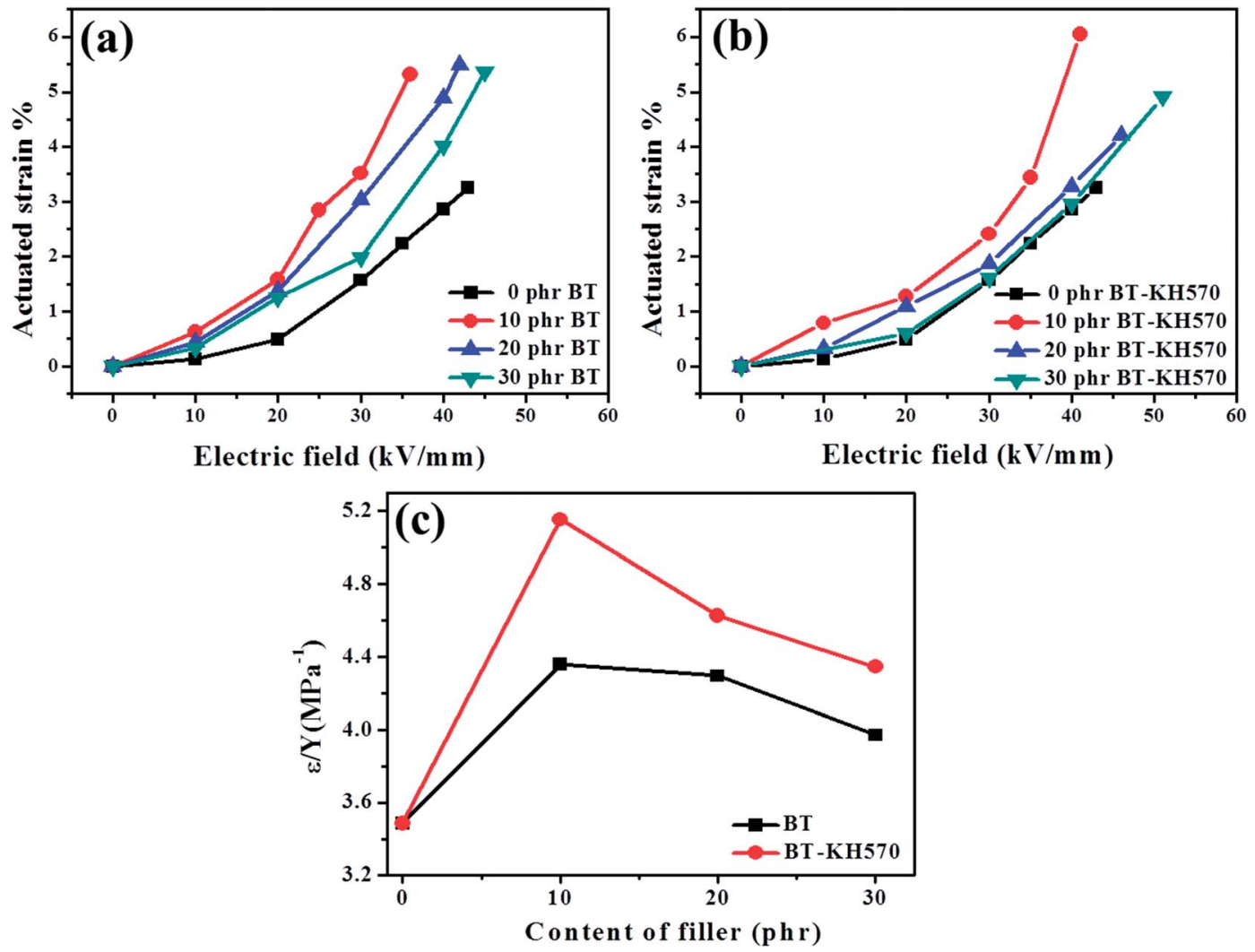

Fig. 8 Dependence of actuated strain of BIIR composites filled with different contents of (a) BT and (b) BT-KH570 particles on electric field. (c) Ratio of dielectric constant to elastic modulus versus filler content of BIIR composites filled with different contents of BT and BT-KH570 particles.

\subsection{Effect of plasticization}

3.2.1 Morphology of dielectric composites. Fig. 9 shows the SEM images of fracture surfaces of $10 \mathrm{phr}$ BT-KH570/BIIR composite filled with (a) 10 phr TBP and (b) 30 phr TBP. From Fig. 9(a) and (b), we can find that the concentration of BTKH570 in $30 \mathrm{phr}$ TBP/BT-KH570/BIIR composite is lower than that of the concentration of BT-KH570 in 10 phr TBP/BT-KH570/ BIIR composite. The low concentration of BT-KH570 is attributed to the TBP in the composites. In addition, the good dispersion of BT-KH570 in TBP/BT-KH570/BIIR composites may be attributed to polar groups $-\mathrm{P}=\mathrm{O}$ on $\mathrm{TBP}$ having compatibility with the ester groups in KH570 and Br groups in BIIR molecular chain. ${ }^{32,33}$
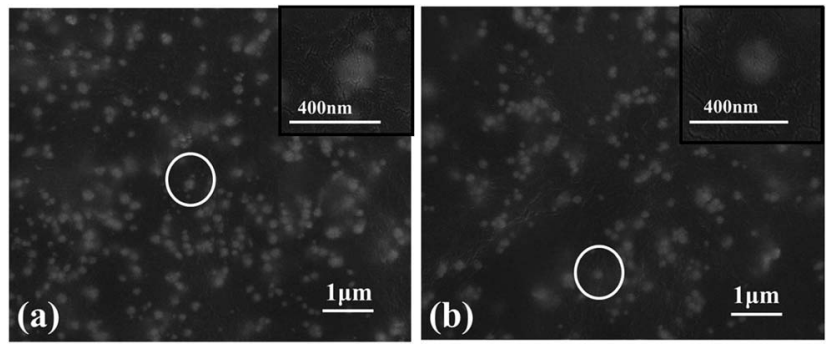

Fig. 9 SEM micrographs of BT-kH570/BIIR composites filled with (a) 10 phr TBP and (b) 30 phr TBP.
3.2.2 Mechanical properties and dielectric properties of dielectric composites. As expected, the plasticizer shows a significant effect on mechanical properties. Fig. 10(a) demonstrates the stress-strain curves of $10 \mathrm{phr}$ BT-KH570/BIIR composite filled with different contents of TBP. From Fig. 10(a), we can see that the elongation at break of TBP/BT-KH570/BIIR composites is much larger than that of BT-KH570/BIIR composite. This is mainly because the low molecular plasticizer weakens the force of the molecular chain. ${ }^{42}$ When more plasticizer is added into the composites, the elongation at break largely increases. The low molecular plasticizer improves the distance between molecular chains, fluidity, and softness of BTKH570/BIIR composites. The elastic modulus of the BT-KH570/ BIIR composites filled with different contents of TBP is shown in Fig. 10(b). From Fig. 10(b), we find that the elastic modulus of composites decreases largely with the addition of TBP, which can be ascribed to the addition of plasticizer TBP destroying the network structure of the fillers and reducing the force in the molecular chains. The lowest elastic modulus is $0.15 \mathrm{MPa}$, which represents a decrease of $55 \%$ in comparison with the BTKH570/BIIR composite without TBP. ${ }^{\mathbf{4 3 , 4 4}}$ The low elastic modulus leads to a high electromechanical sensitivity $\beta$.

Fig. 10(c) demonstrates the frequency dependence of the dielectric constant of $10 \mathrm{phr}$ BT-KH570/BIIR composite filled with different contents of TBP. From Fig. 10(c), we can see that the dielectric constant improves with the increasing content of 

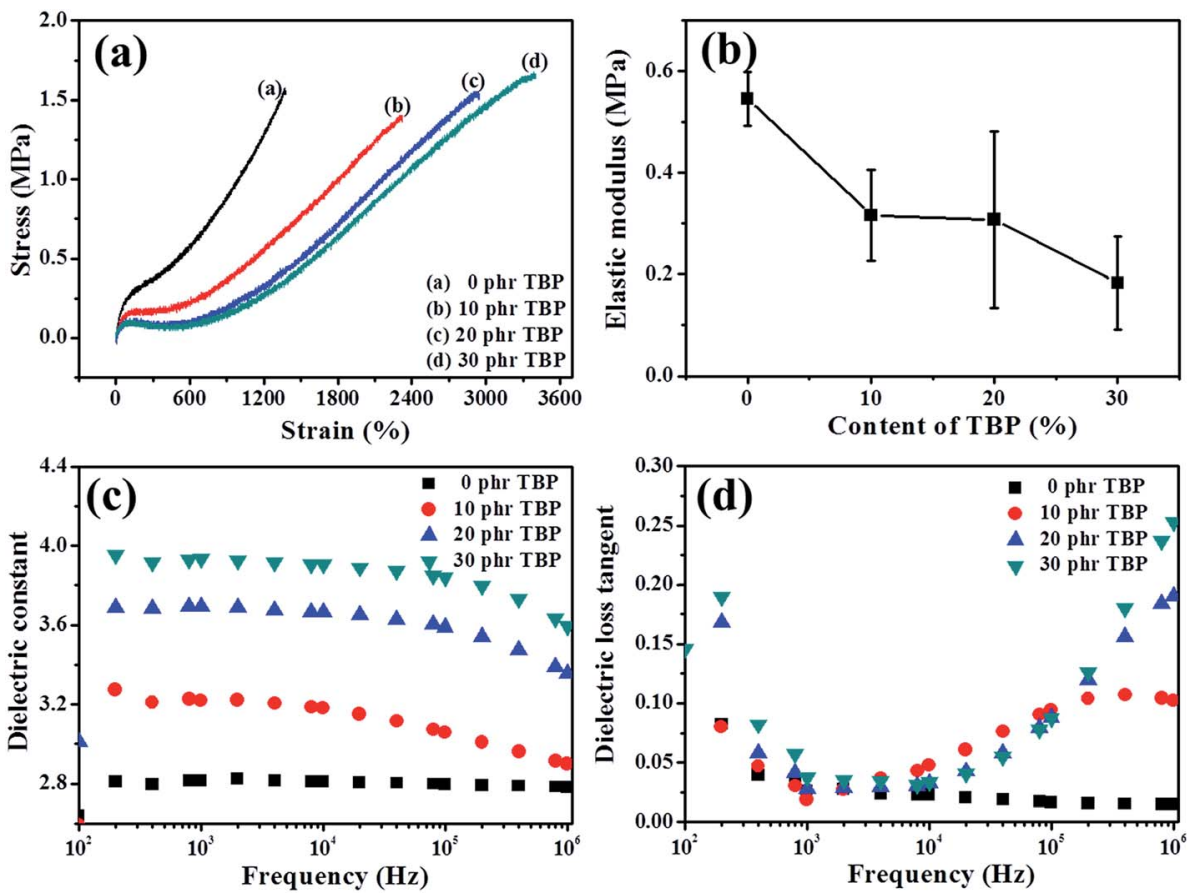

Fig. 10 (a) Stress-strain curves of BT-KH570/BIIR composites filled with different contents of TBP. (b) Elastic modulus of BT-KH570/BIIR composites filled with different contents of TBP. Frequency dependence of (c) dielectric constant and (d) dielectric loss tangent of BT-KH570/ BIIR composites at different contents of TBP.

TBP at the same frequency. With the increasing content of polar plasticizer TBP, free electrons gather at the interface under the electric field, leading to an increase in the dielectric constant. Fig. 10(d) shows the dielectric loss of BT-KH570/BIIR composite with different contents of TBP. From Fig. 10(d), we can find that the dielectric loss shows an evident change from $10^{2}$ to $10^{6} \mathrm{~Hz}$. However, the dielectric loss is lower than 0.05 at $1 \mathrm{kHz}$, which shows that the energy dissipation of DEAs is very little.

3.2.3 Actuated strain of dielectric composites. As shown in Fig. 11(a), the actuated strain of BT-KH570/BIIR composite with different TBP content is plotted against the electric field. From Fig. 11(a), the actuated strain of the composites increases with increasing electric field. The more plasticizer is added into the BT-KH570/BIIR composite, the larger actuated strain of the composites is obtained at the same electric field. ${ }^{45}$ Such a phenomenon can be explained by the destruction of the molecular chain force of the dielectric elastomer material, which leads to the decrease of elastic modulus. As a result, the electromechanical sensitivity $\beta$ increases with the increasing content of TBP (shown in Fig. 11(b)). ${ }^{46}$ The largest actuated strain of $30 \mathrm{phr}$ of TBP/BT-KH570/BIIR composite increases to $4.46 \%$ at $22.5 \mathrm{kV} \mathrm{mm}^{-1}$, which is approximately $500 \%$ times that of pure BIIR. In addition, we compared the electromechanical performance of BIIR composite with that of other dielectric elastomers, and the results are displayed in Table 3. Upon comparison, we could see that the electromechanical sensitivity $\beta$ and actuated strain at $22.5 \mathrm{kV} \mathrm{mm}^{-1}$ of $30 \mathrm{phr}$ TBP/BT-KH570/BIIR are relatively higher than that of other DEA materials listed in Table 3 under the conditions of no prestrain.
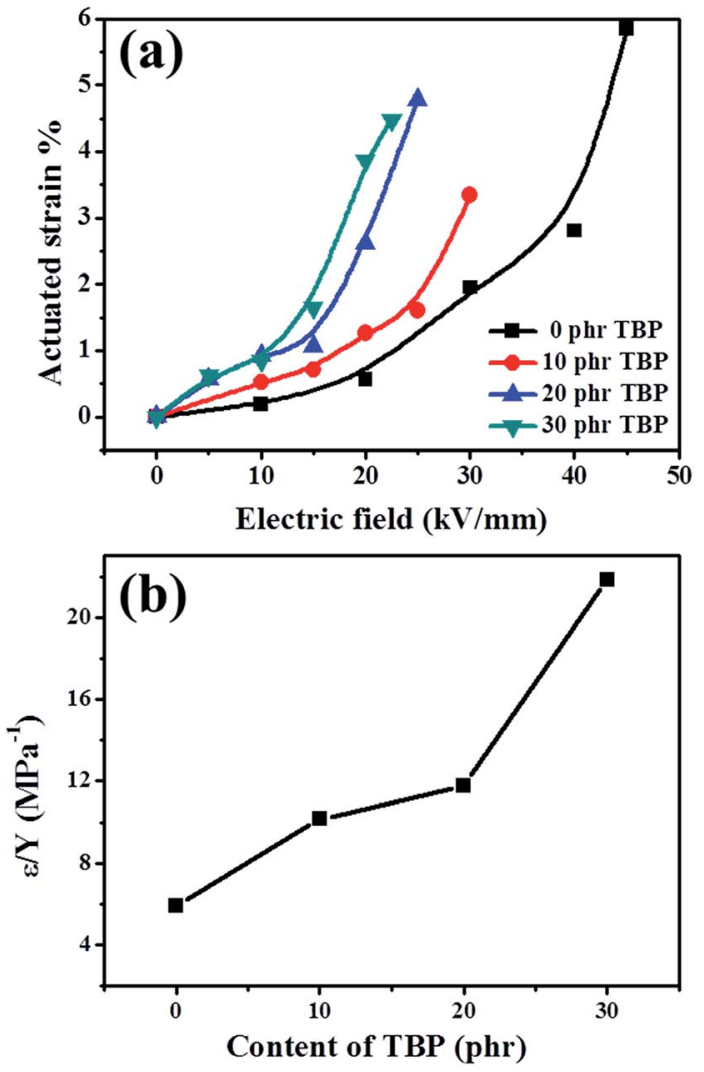

Fig. 11 (a) Dependence of actuated strain of BT-KH570/BIIR composites filled with different contents of TBP. (b) The ratio of dielectric constant to elastic modulus versus filler content of BTKH570/BIIR composites filled with different contents of TBP. 
Table 3 Comparison of the electromechanical properties for various advanced dielectric elastomers reported in the literature

\begin{tabular}{|c|c|c|c|c|c|}
\hline Samples & $\begin{array}{l}\text { Dielectric constant } \\
\text { at } 10^{3} \mathrm{~Hz}\end{array}$ & $\begin{array}{l}\text { Electromechanical sensitivity } \\
\beta \text { at } 10^{3} \mathrm{~Hz}, \mathrm{MPa}^{-1}\end{array}$ & $\begin{array}{l}\text { Maximum planar } \\
\text { actuated } \operatorname{strain}^{b}, \%\end{array}$ & $\begin{array}{l}\text { Actuated } \operatorname{strain}^{b} \\
\text { at } 22.5, \mathrm{kV} \mathrm{mm}^{-1}\end{array}$ & $\begin{array}{l}\text { Breakdown } \\
\text { strength }^{b}, \mathrm{kV} \mathrm{mm} \\
\end{array}$ \\
\hline Acrylic (VHB 4910) ${ }^{a}$ & 4.7 & 10.5 & 158 & - & 412 \\
\hline $10 \mathrm{wt} \% \mathrm{BT} / \mathrm{SR}^{a 48}$ & 4.5 & - & 0.12 & $<0.12$ & 25 \\
\hline $30 \mathrm{phr} \mathrm{DOP} / \mathrm{TiO}_{2} / \mathrm{NBR}^{49}$ & 10.25 & 13.6 & 3.0 & $<3$ & 20 \\
\hline $35 \mathrm{wt} \% \mathrm{TiO}_{2} / \mathrm{XLR}^{50}$ & 4.7 & 16 & 10 & $<2$ & 51 \\
\hline PTMDMT1 (ref. 20) & 3.6 & - & 0.82 & $<0.2$ & 60 \\
\hline 14PANI/15PolyCuPc/85PU & 90 & 0.64 & 9.5 & - & 19 \\
\hline Pure BIIR & 2.63 & 3.3 & 3.2 & 1 & 40 \\
\hline $30 \mathrm{phr}$ TBP/BT-KH570/BIIR & 4.0 & 22.1 & 4.6 & 4.6 & 22.5 \\
\hline
\end{tabular}

${ }^{a}$ The acrylic, HS3 silicone, and $10 \mathrm{wt} \% \mathrm{BT} / \mathrm{SR}$ are $300 \%, 68 \%$, and $160 \%$ prestrained respectively, other data collected herein are with no prestrain. ${ }^{b}$ Estimated from graphical data in cited reference, when no tabulated results were provided.

The chemical structures and molecular interactions of TBP and BIIR composites were characterized by FTIR, as shown in Fig. S1. $\dagger$ We can see the characteristic absorption peaks of 659 $\mathrm{cm}^{-1}, 1544 \mathrm{~cm}^{-1}$, and $1732 \mathrm{~cm}^{-1}$ in Fig. S1(a), $\dagger$ which represent $\mathrm{C}-\mathrm{Br}, \mathrm{C}=\mathrm{C}$, and $\mathrm{C}=\mathrm{O}$ in BT-KH570/BIIR composite, respectively. The positions of $\mathrm{C}=\mathrm{O}$ from $\mathrm{KH} 570$ are shifted towards higher wavenumber, which may be the presence of molecular interactions between BT-KH570 and BIIR chains as shown in Fig. S1(b). $\dagger$ We can also see that the characteristic absorption peaks of TBP appear at $1028 \mathrm{~cm}^{-1}, 1030 \mathrm{~cm}^{-1}$, and $1281 \mathrm{~cm}^{-1}$, which represent $\mathrm{P}=\mathrm{O}$ and $\mathrm{C}-\mathrm{O}-\mathrm{P}$ stretching mode, respectively. ${ }^{47}$ These characteristic peaks of TBP also appear in TBP/BT-KH570/BIIR composite, which can prove the existence of TBP.

\section{Conclusion}

As the BT-KH570 particles exhibit good compatibility with the BIIR matrix, BT-KH570/BIIR composites demonstrate better dielectric properties, mechanical properties, and electromechanical properties than that of BT/BIIR composites. The BIIR composite filled with 10 phr BT-KH570 had the largest strain. To further improve actuated strain of $10 \mathrm{phr}$ BT-KH570/BIIR composite, TBP plasticizer was added into BT-KH570/BIIR composites to reduce the elastic modulus and increase the dielectric constant of the elastomer. Furthermore, an actuated strain of $4.46 \%$ at the electric field of $22.5 \mathrm{kV} \mathrm{mm}^{-1}$ was obtained, which represented an increase of $500 \%$ times in comparison to that of pure BIIR. The results indicated that the TBP/BT-KH570/BIIR composite had a significantly larger improvement on actuated strain performance than that of pure BIIR composite.

\section{Acknowledgements}

This study was supported by the National Natural Science Foundation of China (No. 51503019, No. 51373026, No. 51573020, and No. 21501012), Beijing Natural Science Foundation (No. 2162014),
Beijing Science and Technology Project of Beijing Municipal Education Commission (KM201710017005).

\section{Notes and references}

1 M. Gei, S. Colonnelli and R. Springhetti, Int. J. Solids Struct., 2014, 51, 848-860.

2 W. Hu, S. Zhang, X. Niu, C. Liu and Q. Pei, J. Mater. Chem. C, 2014, 2, 1658-1666.

3 L. J. Romasanta, M. A. Lopez-Manchado and R. Verdejo, Prog. Polym. Sci., 2015, 51, 188-211.

4 P. H. Vargantwar, K. E. Roskov, T. K. Ghosh and R. J. Spontak, Macromol. Rapid Commun., 2012, 33, 61-68.

5 S. K. Yadav, I. J. Kim, H. J. Kim, J. Kim, S. M. Hong and M. K. Chong, J. Mater. Chem., 2013, 1, 5463-5470.

6 T. Chen, J. Qiu, K. Zhu, X. He, X. Kang and E. L. Dong, Mater. Lett., 2014, 128, 19-22.

7 G. Mathew, J. M. Rhee, C. Nah and D. J. Leo, Polym. Eng. Sci., 2006, 46, 1455-1460.

8 M. Razzaghikashani, Proc. SPIE, 2010, 7642, 579-595.

9 C. Racles, A. Bele, M. Dascalu, V. E. Musteata, C. D. Varganici, D. Ionita, S. Vlad, M. Cazacu, S. J. Dünki and D. M. Opris, $R S C A d v$., 2015, 5, 58428-58438.

10 T. Chen, J. Qiu, K. Zhu and J. Li, Mater. Des., 2016, 90, 10691076.

11 X. Qi, S. Zheng and F. Dai, Appl. Polym. Sci., 2007, 33, 43904.

12 J. J. Sheng, H. L. Chen and B. Li, J. Funct. Mater. Devices, 2011, 17, 549-554.

13 W. Zhou, Y. Gong, L. Tu, L. Xu, W. Zhao, J. Cai, Y. Zhang and A. Zhou, J. Alloys Compd., 2017, 693, 1-8.

14 S. K. Yadav, S. S. Mahapatra, J. W. Cho, H. C. Park and J. Y. Lee, Fibers Polym., 2009, 10, 756-760.

15 M. P. Silva, C. M. Costa, V. Sencadas, A. J. Paleo and S. Lanceros-Méndez, J. Polym. Res., 2011, 18, 1451-1457.

16 G. Gallone, F. Carpi, D. D. Rossi, G. Levita and A. Marchetti, Mater. Sci. Eng., C, 2007, 27, 110-116.

17 Z. M. Dang, H. P. Xu and H. Y. Wang, Appl. Phys. Lett., 2007, 90, 012901-012903. 
18 Y. Jang, T. Kato, T. Ueki and T. Hirai, Sens. Actuators, A, 2011, 168, 300-306.

19 H. Sun, C. Jiang, N. Ning, L. Zhang, M. Tian and S. Yuan, Polym. Chem., 2016, 7, 4072-4080.

20 K. Y. Cho, A. Cho, H. J. Kim, S. H. Park, M. K. Chong, Y. J. Kwark, H. G. Yoon, S. S. Hwang and K. Y. Baek, Polym. Chem., 2016, 7, 7391-7399.

21 N. K. Dutta and D. K. Tripathy, Polym. Test., 1990, 9, 3-13.

22 T. Zhou, J. W. Zha, R. Y. Cui, B. H. Fan, J. K. Yuan and Z. M. Dang, ACS Appl. Mater. Interfaces, 2011, 3, 2184-2188.

23 Z. M. Dang, H. Y. Wang, Y. H. Zhang and J. Q. Qi, Macromol. Rapid Commun., 2010, 26, 1185-1189.

24 L. Jiang, A. Betts, D. Kennedy and S. Jerrams, Mater. Des., 2015, 85, 733-742.

25 Z. M. Dang, Y. J. Xia, J. W. Zha, J. K. Yuan and J. Bai, Mater. Lett., 2011, 65, 3430-3432.

26 D. Yang, F. Ge, M. Tian, N. Ning, L. Zhang, C. Zhao, K. Ito, T. Nishi, H. Wang and Y. Luan, J. Mater. Chem. A, 2015, 3, 9468-9479.

27 Y. Min, Y. Fang, X. Huang, Y. Zhu, W. Li, J. Yuan, L. Tan, S. Wang and Z. Wu, Appl. Surf. Sci., 2015, 346, 497-502.

28 G. H. Wu, S. Q. Liu, X. Y. Wu and X. M. Ding, J. Polym. Res., 2016, 23, 1-8.

29 D. Yang, M. Ruan, S. Huang, Y. Wu, S. Li, H. Wang, X. Ao, Y. Liang, W. Guo and L. Zhang, RSC Adv., 2016, 6, 9017290183.

30 M. X. Shi, M. Zhang, Z. X. Huang and Y. Qin, Adv. Mater. Res., 2010, 160-162, 359-363.

31 D. Yang, M. Tian, D. Li, W. Wang, F. Ge and L. Zhang, J. Mater. Chem. A, 2013, 1, 12276-12284.

32 G. B. Shah, M. Fuzail and J. Anwar, J. Appl. Polym. Sci., 2004, 92, 3796-3803.

33 D. Yang, M. Ruan, S. Huang, Y. Wu, S. Li, H. Wang, Y. Shang, B. Li, W. Guo and L. Zhang, J. Mater. Chem. C, 2016, 4, 77247734.

34 D. Yang, M. Tian, H. Kang, Y. Dong, H. Liu, Y. Yu and L. Zhang, Mater. Lett., 2012, 76, 229-232.

35 G. X. Qiu, F. Raue and G. W. Ehrenstein, J. Appl. Polym. Sci., 2010, 83, 3029-3035.
36 J. Wang, C. Wu, R. Liu and S. Li, Polym. J., 2014, 46, 285292.

37 L. Duan, G. L. Wang, Y. Y. Zhang, Y. N. Zhang, Y. Y. Wei, Z. F. Wang and M. Zhang, Polym. Compos., 2016, DOI: 10.1002/pc.23986.

38 R. Pelrine, R. Kornbluh, Q. Pei and J. Joseph, Science, 2000, 287, 836-839.

39 R. Pelrine, R. Kornbluh, J. Joseph, R. Heydt, Q. Pei and S. Chiba, Mater. Sci. Eng., C, 2000, 11, 89-100.

40 H. Lee, S. M. Dellatore, W. M. Miller and P. B. Messersmith, Science, 2007, 318, 426-430.

41 Y. Song, Y. Shen, H. Liu, Y. Lin, M. Li and C. W. Nan, J. Mater. Chem., 2012, 22, 8063-8068.

42 D. Yang, M. Tian, Y. Dong, H. Liu, Y. Yu and L. Zhang, Smart Mater. Struct., 2012, 21, 035017.

43 Y. Liu, L. Liu, Z. Zhang and J. Leng, Smart Mater. Struct., 2009, 18, 95024.

44 F. Galantini, F. Carpi and G. Gallone, Smart Mater. Struct., 2013, 22, 104020.

45 W. Lei, R. Wang, D. Yang, G. Hou, X. Zhou, H. Qiao, W. Wang, M. Tian and L. Zhang, RSC Adv., 2015, 5, 4742947438.

46 X. Niu, H. Stoyanov, W. Hu, R. Leo, P. Brochu and Q. Pei, J. Polym. Sci., Polym. Phys. Ed., 2012, 51, 197-206.

47 S. R. C. Vivekchand, K. C. Kam, G. Gundiah, A. Govindaraj, A. K. Cheetham and C. N. R. Rao, J. Mater. Chem., 2005, 15, 4922-4927.

48 L. Jiang, A. Betts, D. Kennedy and S. Jerrams, Mater. Sci. Eng., C, 2015, 49, 754-760.

49 H. Chuc Nguyen, V. Thuy Doan, J. K. Park, J. C. Koo, Y. Lee, J. Nam and H. Ryeol Choi, Smart Mater. Struct., 2008, 18, 015006.

50 L. Yu and A. L. Skov, Int. J. Smart Nano Mater., 2015, 6, 268289.

51 I. J. Kim, K. Min, H. Park, S. M. Hong, W. N. Kim, S. H. Kang and M. K. Chong, J. Appl. Polym. Sci., 2014, 131, 596-602.

52 H. Stoyanov, M. Kollosche, D. N. Mccarthy and G. Kofod, J. Mater. Chem., 2010, 20, 7558-7564. 